\title{
Avaliação estrutural do polo posterior em pacientes com doença de Behçet
}

\author{
Structural evaluation of posterior pole in patients with Behçet's disease \\ Ticiana Paula Resende CorrêA ${ }^{1}$, Tiago Eugênio Faria e Arantes², Verônica Castro Lima², Cristina Muccioli²
}

\section{RESUMO}

Objetivo: Avaliar achados demográficos, de exame ocular, alterações vasculares e estruturais por meio de angiografias com fluoresceína e indocianina verde e de tomografia de coerência óptica em retina e coroide em pacientes com doença de Behçet com controle clínico.

Método: Revisão de prontuários de 16 pacientes com doença de Behçet em fase inativa da doença. Foram submetidos a exame oftalmológico, angiografias com fluoresceína e indocianina e tomografia de coerência óptica e divididos em dois grupos de acordo com o tempo de doença.

Resultados: Avaliou-se 13 pacientes do sexo feminino e 3 do sexo masculino. Os principais achados de exame ocular foram estreitamento vascular, catarata, atrofia do disco óptico e membrana epirretiniana macular. Sessenta e dois e meio por cento dos pacientes estavam com acuidade visual igual ou melhor que 0,1 . Os principais achados na angiografia com fluoresceína foram vazamento capilar e impregnação da parede vascular, na angiografia com indocianina verde foram lesões hipofluorescentes bem definidas e na tomografia de coerência óptica foram membrana epirretiniana e atrofia retiniana. Analisando a acuidade visual, não se encontrou diferença estatística entre os parâmetros de sexo, tempo de doença, presença de edema retiniano na tomografia de coerência óptica ou na angiografia com fluoresceína. O aumento da espessura macular não se correlacionou positivamente com a idade, tempo de doença ou com a acuidade visual. O encontro de afinamento vascular na angiografia com fluoresceína correlacionou-se com maior duração da doença $(p=0,033)$. Os demais achados dos exames não se correlacionaram com o tempo de doença.

Conclusão: Os exames de angiografias com fluoresceína e indocianina verde e tomografia de coerência óptica fornecem dados importantes do acometimento do polo posterior na doença de Behçet. Apesar do aparente controle clínico, esses exames podem evidenciar atividade inflamatória persistente, a qual ocasiona progressão da perda visual e significante número de pacientes com cegueira legal.

Descritores:Síndrome de Behçet/diagnóstico; Uveíte; Coroide/irrigação sanguínea; Retina/irrigação sanguínea; Angiofluoresceinografia; Verde de indocianina/uso diagnóstico; Tomografia de coerência óptica

\begin{abstract}
Purpose: To evaluate demographic findings, ocular manifestations, vascular and morphological changes by fluorescein and indocyanine green angiography and optical coherence tomography of retina and choroid in cases of ocular Behçet's disease.

Methods: Medical records were reviewed to obtain data from 16 patients with Behçet's disease who were in inactive phase. All patients underwent ophthalmic examination, simultaneous fluorescein and indocyanine green angiography and optical coherence tomography. They were also divided into two groups according to disease duration.

Results: Thirteen females and three males were evaluated. The main findings of ocular exams were narrowing of vessels, cataract, atrophy of the optic disc and macular epiretinal membrane. $62.5 \%$ had visual acuity equal to or better than 0.1 . The main findings of fluorescein angiography were capillary leak and impregnation of the vascular wall. The most important findings of indocyanine green angiography were hypofluorescent well defined lesions. The main findings of optical coherence tomography were macular epiretinal membrane and retinal atrophy. Analyzing the visual acuity, no statistical correlation was found between gender, disease duration or presence of retinal edema in fluorescein angiography or optical coherence tomography. The increase in macular thickness was not correlated positively with patient age, disease duration or with the visual acuity. Vascular narrowing seen on fluorescein angiography was correlated with longer duration of illness $(p=0.033)$. The remaining test findings were not correlated with disease duration.

Conclusion: Fluorescein and indocyanine green angiography and optical coherence tomography are valuable tools to assess the posterior pole in Behçet's disease. Despite the apparent clinical control, these tests may show persistent inflammatory activity, which causes progression of visual loss and a significant number of patients with legal blindness.
\end{abstract}

Keywords: Behçet syndrome/diagnosis; Uveitis; Choroid/blood supply; Retina/blood supply; Fluorescein angiography; Indocyaninegreen/diagnosticuse; Tomography, optical coherence

\section{INTRODUÇÃO}

A doença de Behçet (DB), descrita em 1937 pelo dermatologista turco Hulusi Behçet, é uma desordem crônica multissistêmica com inflamação recorrente de etiologia desconhecida, provavelmente mediada por imunocomplexos ${ }^{(1)}$ e de predisposição genética devido à associação com o grupo de HLA B 51. Apresenta maior prevalência em países do Mediterrâneo e Japão.

A doença é diagnosticada pela presença de úlceras orais recorrentes acrescidas de pelo menos dois dos seguintes sinais: úlceras genitais, alteração ocular como uveíte recorrente, alterações de pele ou teste de patergia positivo ${ }^{(2)}$. O estudo histopatológico demonstra vasculite obliterativa e necrosante de artérias e veias ${ }^{(3)}$.

O envolvimento ocular está presente em 70 a 90\% dos pacientes. Afeta ambos os polos anterior e posterior, caracterizado por uveíte anterior com hipópio, vasculite oclusiva retiniana, vitreíte, hemorragia, edema macular, infiltrados e exsudatos algodonosos, neovasos de retina e de nervo óptico, isquemia retiniana e macular. O descolamento de retina tracional e as atrofias retiniana e de
Funding: No specific financial support was available for this study.

Disclosure of potential conflicts of interest: T.P.R.Corrêa, None; T.E.Faria e Arantes, None; V.C.Lima, None; C.Muccioli, None.

Correspondence address: Ticiana Paula Resende Corrêa. Avenida João Naves de Ávila, 888 Apto. 401 - Uberlândia (MG) - 38400-042 - Brazil - E-mail: tici_p@yahoo.com.br

Número do Projeto e Instituição responsável pelo parecer do Comitê de Ética em Pesquisa: № 1661/10 
nervo óptico são alterações avançadas da doença. Atinge o polo posterior em 50 a $93 \%$ dos casos. A atividade inflamatória persistente leva à perda visual, determinando cegueira em cerca de 1/3 dos pacientes ${ }^{(4-6)}$.

Como métodos de diagnóstico e seguimento da doença em polo posterior, temos o estudo da vasculatura através da angiografia com fluoresceína (AF) e indocianina verde (ICV), e a análise estrutural das camadas retinianas por meio da tomografia de coerência óptica (OCT).

A angiografia com fluoresceína estuda os vasos retinianos e seus capilares, fornecendo informações já validadas para a investigação e acompanhamento das alterações oculares por vasculite ${ }^{(7)}$. Detecta também edema macular, oclusões e neovasos de retina e alterações no disco óptico. Porém não fornece informações detalhadas sobre a circulação da coroide. Para isso tem-se a angiografia com indocianina verde, que avalia o envolvimento inflamatório dos vasos coroideanos ${ }^{(8,9)}$. Ambas são complementares, sendo utilizadas simultaneamente para melhor estudo das alterações inflamatórias e sequelares dessa doença ${ }^{(9-11)}$, com desvantagem de serem exames invasivos.

As alterações estruturais causadas pela doença são detectadas pela OCT, que utiliza técnica não invasiva para fornecer imagens de cortes in vivo das camadas retinianas com alta resolução. Tem como desvantagens a necessidade de claridade de meios, análise restrita ao polo posterior e profundidade de penetração limitada.

O objetivo do estudo é demonstrar achados demográficos, de exame físico, alterações vasculares e estruturais de AF, ICV e OCT em retina e coroide na DB em pacientes controlados clinicamente.

\section{MÉTODOS}

Vinte e sete olhos de 16 pacientes com diagnóstico de DB clinicamente inativa foram incluídos no estudo. Todos preencheram os critérios de diagnóstico do International Study Group for Behçet Disease $^{(2)}$

Foram revisados prontuários para levantamento dos seguintes dados: idade, sexo, tempo de doença, manifestações extraoculares, bilateralidade e medicações em uso. Submeteram-se no momento de inclusão no estudo a medida da acuidade visual (AV) com uso da tabela do ETDRS, exame biomicroscópico em lâmpada de fenda, tonometria com tonômetro de Goldmann e exame de oftalmoscopia indireta. Os pacientes ainda foram divididos em dois grupos para análise da influência do tempo de doença nas variáveis estudadas: menos de 10 anos de doença (8 pacientes, 13 olhos) e mais de 10 anos de doença (8 pacientes, 14 olhos).

Definimos como fase inativa da doença o quadro clínico com aparente ausência de inflamação anterior e posterior: ausência de reação de câmara anterior ou precipitados ceráticos recentes, hipópio, retinite, vasculite, vitreíte, papilite, edema macular, etc.

O estudo foi aprovado pelo Comitê de Ética e Pesquisa da Universidade Federal de São Paulo. Todos os pacientes assinaram o Termo de Consentimento Informado.

As angiografias e a OCT foram realizadas na mesma sessão em todos os pacientes no período de dezembro de 2009 a dezembro de 2010. Foram excluídos do estudo 5 olhos de 5 pacientes devido à qualidade insatisfatória dos exames por opacidade de meios.

Utilizando o aparelho HRA (Heidelberg Engineering Inc., Heidelberg, Alemanha) as angiografias foram feitas com fluoresceína e indocianina. Inicialmente, foram injetados $5 \mathrm{ml}$ de fluoresceína sódica a 10\% na veia cubital, com documentação por fotos nas fases inicial (2 minutos), intermediária (4 a 7 minutos) e tardia (10 minutos). Imediatamente após a AF, foram injetadas $25 \mathrm{mg}$ de indocianina verde diluída, sendo documentadas as fases inicial (0 a 3 minutos), intermediária (8 a 12 minutos) e tardia (>35 minutos).

A OCT foi realizada em sequência usando o aparelho OCT Spectrallis (Heidelberg Engineering Inc., Heidelberg, Alemanha) com avaliação da região macular com múltiplos cortes e mapa de espessura macular.
Os exames foram revisados e analisados por duas médicas, que ignoravam a acuidade visual dos pacientes e qualquer outro dado relevante.

Utilizou-se a estatística descritiva para a caracterização da amostra. As variáveis contínuas foram expressas como média \pm desvio padrão e as variáveis categóricas foram expressas por frequência absoluta ( $\mathrm{n}$ ) e relativa (\%).

A distribuição dos dados foi analisada por meio do teste de normalidade de Lilliefors. As comparações entre médias de duas amostras foram feitas utilizando-se teste t de Student. As análises de correlação foram realizadas utilizando o coeficiente de Spearman. Foi utilizado o teste do Qui-quadrado para correlação das variáveis qualitativas. Quando a frequência esperada era menor do que 5, a significância estatística foi avaliada utilizando-se o método de Monte Carlo com 10.000 simulações.

Todos os testes foram considerados significativos quando $p<0,05$.

A análise estatística foi realizada usando o software Statistical Package for the Social Sciences (SPSS para Windows, versão 17.0; Chicago; IL) ${ }^{(12)}$.

\section{RESULTADOS}

Analisou-se dados de prontuário e exames de 27 olhos de 16 pacientes. Treze pacientes eram do sexo feminino e 3 do sexo masculino. A média de idade foi de 38,75 anos (27 a 64 anos), de duração da doença no momento dos exames foi de 14 anos (3 a 30 anos) e de idade de início de sintomas foi de 24,75 anos (14 a 32 anos). Cem por cento dos pacientes apresentaram úlceras orais, 62,5\% úlceras genitais, 56,3\% manifestações dermatológicas e 50\% artrite desde o início da doença. Três pacientes estavam sem medicação sistêmica, 37,5\% usavam prednisona, 31\% azatioprina e $31 \%$ ciclosporina. Sessenta e dois e meio por cento apresentavam no momento do exame AV igual ou melhor que 0,1, com média de AV de 0,34 (1 a vultos). Os dados de exame físico estão descritos na tabela 1

Não houve diferença estatística entre os dois sexos quanto aos achados demográficos ou de exame físico.

Os achados nos exames de AF e ICV e OCT estão resumidos nas tabelas 2, 3 e 4 .

Correlacionando a presença de lesões concomitantemente encontradas na AF e na ICV, verificou-se que:

- 29,6\% dos olhos apresentavam lesões hipofluorescentes na ICV ausentes na AF;

- 7,4\% apresentavam lesões hipofluorescentes na AF ausentes na ICV;

- 22,2\% apresentavam lesões hipofluorescentes concomitantes na ICV e AF:

Tabela 1. Características do exame oftalmológico

\begin{tabular}{lc}
\hline Achados & Prevalência (\%) \\
\hline Estreitamento vascular & $70,4 \%$ \\
Catarata & $40,7 \%$ \\
Atrofia do disco optico & $37,0 \%$ \\
Membrana epirretiniana & $37,0 \%$ \\
Pseudofácico & $18,5 \%$ \\
Cicatriz retinite & $14,8 \%$ \\
Pseudoretinose & $12,5 \%$ \\
Glaucoma & $11,1 \%$ \\
Buraco macular & $6,3 \%$ \\
Vasculite & $6,3 \%$ \\
Bolha filtrante & $6,3 \%$ \\
Edema macular & $0 \%$ \\
Neovasos & $0 \%$ \\
\hline
\end{tabular}


Tabela 2. Achados da angiografia com fluoresceína

\begin{tabular}{lc}
\hline Achados & Prevalência (\%) \\
\hline Vazamento capilar & $52,0 \%$ \\
Impregnação parede vascular & $33,0 \%$ \\
Extravasamento vascular & $22,0 \%$ \\
Estravasamento peridiscal & $22,0 \%$ \\
Isquemia macular & $18,5 \%$ \\
Vazamento perifoveal & $15,0 \%$ \\
Cicatrizcoriorretinite & $7,4 \%$ \\
Neovasos papila/retina & $0 \%$ \\
Remodelamento vascular & $0 \%$ \\
Descolamento epitelio pigmentar & $0 \%$ \\
Dobras coroide & $0 \%$ \\
\hline
\end{tabular}

Tabela 3. Achados da angiografia com indocianina verde

\begin{tabular}{lc}
\hline Achados & Prevalência (\%) \\
\hline Lesão hipofluorescente bem definida & $55,6 \%$ \\
Lesão hiperfluorescente & $18,5 \%$ \\
Lesão hipofluorescente pouco definida & $0 \%$ \\
Vazamento veias & $0 \%$ \\
Vazamento coriocapilar & $0 \%$ \\
Preenchimento irregular & $0 \%$ \\
\hline
\end{tabular}

Tabela 4. Achados da tomografia de coerência óptica

\begin{tabular}{lc}
\hline Achados & Prevalência (\%) \\
\hline Membrana epirretiniana & $37,0 \%$ \\
Atrofia retina & $33,0 \%$ \\
Líquido intrarretiniano & $14,8 \%$ \\
Destruição camadas externas & $14,8 \%$ \\
Descolamento vítreo posterior & $3,7 \%$ \\
Buraco macular & $3,7 \%$ \\
Pucker macular & $0 \%$ \\
Vítreosquise & $0 \%$ \\
Tração vítrea & $0 \%$ \\
\hline
\end{tabular}

- 3,7\% apresentavam lesões hiperfluorescentes na ICV ausentes na AF;

- 40,7\% apresentavam lesões hiperfluorescentes na AF ausentes na ICV;

- $26 \%$ apresentavam lesões hiperfluorescentes concomitantes na ICV e AF.

Ao analisar o edema macular, 4 olhos tinham vazamento perifoveal na $\mathrm{AF}$ e 4 olhos tinham líquido intrarretiniano na OCT. O edema foi encontrado simultaneamente nos dois exames (AF e OCT) em 3 olhos (75\%), somente na AF em 1 olho e somente na OCT em 1 olho. Vinte e três olhos $(85,2 \%)$ não tinham edema em nenhum dos dois exames.

Analisando a AV dos pacientes, não houve diferença estatística entre os parâmetros de sexo, tempo de doença $(<10$ anos e $>10$ anos), presença de edema retiniano na OCT ou na AF.

O aumento da espessura macular não se correlacionou positivamente com a idade dos pacientes, tempo de doença ou com a AV.

Em relação ao tempo de doença, não foi encontrada diferença estatística quanto aos achados de exame físico, OCT, ICV e quanto à concordância de achados de lesões hiperfluorescentes e hipofluorescentes na AF e ICV. Quanto aos achados de AF, o encontro de afinamento vascular correlacionou-se com maior duração da doença $(p=0,033)$. Os demais achados de AF não se correlacionaram com o tempo de doença.

\section{DISCUSSÃO}

A DB é uma doença crônica com comprometimento ocular na maioria dos pacientes ${ }^{(13)}$. É caracterizada por uveíte difusa e episódios de vasculite oclusiva que causam alterações irreversíveis isquêmicas da retina neurossensorial e baixa acuidade visual (BAV). A progressão da doença, devido ataques de recorrência da doença e mesmo durante seu aparente controle, leva à cegueira legal em grande parte dos pacientes após anos de doença ${ }^{(14)}$. Em nosso estudo encontramos, após em média 14 anos de doença, 38,5\% dos olhos com níveis de AV menor que 0,1. Outros pesquisadores encontraram valores de AV <0,1 em 20,4 a 30,9\% dos pacientes avaliados ${ }^{(4-6,15)}$

A doença tem início na segunda a quarta décadas de vida, podendo variar desde o primeiro ano de vida até a idade avançada. Nesse estudo temos o início da DB com 24,75 anos (em média), variando de 14 a 34 anos.

A predileção pelo sexo masculino varia com a região em estudo(4-6,14,16-19). Nesse estudo, encontrou-se predileção pelo sexo feminino $(81,3 \%)$, descrita também em outros estudos ${ }^{(18-20)}$. Verifica-se pior prognóstico para sexo masculino ${ }^{(4,15)}$, relacionado ao maior índice de panuveíte em homens ${ }^{(5)}$. No presente estudo não houve diferença estatística entre a AV ou manifestações de exame físico entre os dois sexos. Resultado similar foi encontrado em estudo espanhol, que também não encontrou diferença entre os sexos nos parâmetros avaliados ${ }^{(18)}$.

A doença não tem teste laboratorial patognomônico. O diagnóstico é baseado em achados clínicos, agrupados em critérios pelo International Study Group ${ }^{(2)}$. As manifestações mais frequentes em nosso estudo foram as úlceras orais e genitais, o que também foi verificado por outros autores ${ }^{(19,21)}$. Avaliando 49 pacientes brasileiros no período de fevereiro de 1974 a junho de 1990, os autores encontraram aftas orais em $98 \%$, úlceras genitais em $55,1 \%$, lesões dermatológicas em 51\% e lesões articulares em 44,9\%(22). Geralmente a doença ocular é bilateral, característica também confirmada no presente estudo.

As principais complicações da doença que acarretam perda da AV descritas na literatura são catarata, neuropatia óptica glaucomatosa e isquêmica(23), oclusões vasculares e alterações retinianas como edema e atrofia ${ }^{(8,24)}$. No presente estudo verificou-se também como complicações mais frequentes da doença crônica o estreitamento vascular oclusivo, catarata, neuropatias ópticas e membrana epirretiniana. Não se encontrou diferença estatística entre os achados de exame físico em pacientes com menos de 10 anos ou mais de 10 anos de doença.

A retina é o sítio primário da doença, sendo a coriocapilar também afetada. $\mathrm{O}$ estudo da vasculatura retiniana e coroideana e a análise morfológica da retina fornecem dados que corroboram o diagnóstico clínico, auxiliam na detecção de alterações isquêmicas da retina e monitoram o tratamento.

A angiofluoresceinografia é utilizada desde 1960 para identificar alterações vasculares em uveítes. A fluoresceína é ideal para delimitar a rede capilar retiniana ${ }^{(11)}$, porém não evidencia bem lesões coroideanas.

Nesse estudo verificou-se como principais características da AF o vazamento capilar, impregnação da parede vascular, extravasamento vascular - principalmente venoso - extravasamento peridiscal e isquemia macular. Houve correlação significativa entre tempo de doença e estreitamento vascular visível na AF $(p=0,003)$, demonstrando o aumento da incidência e extensão da vasculite oclusiva durante a evolução da doença. Nas demais alterações detectadas na AF não foi encontrada diferença estatística quanto ao tempo de doença (<10 anos e >10 anos). 
Alguns autores demonstraram extravasamento vascular em $50,9 \%$, vazamento perifoveal em $16,1 \%$ e peridiscal em 3,6\% dos olhos estudados ${ }^{(10)}$. Um estudo que avaliou olhos com a doença em fase ativa encontrou extravasamento vascular em 73,5\%, peridiscal em $89,8 \%$, vazamento perifoveal em $63,3 \%$ e isquemia macular em $6,1 \%$ dos olhos avaliados ${ }^{(11)}$. Pacientes chineses apresentaram extravasamento vascular em 76,4\% e capilarite em $97,9 \%$ dos olhos estudados ${ }^{(15)}$.

O uso da ICV é justificado pelo achado de alterações coroideanas em casos de fundoscopia e AF normais. Histopatologicamente há infiltração leucocitária e deposição de imunocomplexos nos vasos da coroide em pacientes com DB ${ }^{(25)}$.

Nesse estudo detectou-se placas hipofluorescentes bem definidas em 55,6\% dos olhos e hiperfluorescentes em 18,5\% dos casos. Não se encontrou diferença estatística entre os achados da ICV e tempo de doença, também não encontrada em outro trabalho com pacientes com doença inativa ${ }^{(9)}$. Avaliando pacientes com doença ativa e inativa, os autores descreveram lesões hipofluorescentes em $35 \%$ e hiperfluorescentes em $66 \%$ dos olhos estudados ${ }^{(8)}$.

Houve concordância dos achados de lesões hipofluorescentes na AF e ICV em 22,2\% dos casos e hiperfluorescentes em $26 \%$. A ICV identificou mais lesões hipofluorescentes que a AF $(29,6 \%)$ e a AF mais lesões hiperfluorescentes (40,7\%).

Autores italianos demonstraram lesões vistas apenas na ICV (e não detectadas na AF) em 19,23\% dos olhos e lesões detectadas apenas na AF em 11,54\% dos olhos ${ }^{(9)}$. Pesquisadores turcos concluíram que $76,8 \%$ das lesões hiperfluorescentes e hipofluorescentes na ICV não foram vistas na $A^{(10)}$. Um outro estudo encontrou $16,3 \%$ dos olhos com lesões em AF não vistas na ICV e somente $2 \%$ dos olhos com lesões visíveis apenas na ICV(11).

A evolução do dano isquêmico retiniano provoca alterações morfológicas na retina neurossensorial. Essas alterações são avaliadas com uso da OCT, técnica tão eficaz quanto à AF para avaliar o líquido intrarretiniano, sendo superior em demonstrar a distribuição desse fluido e alterações estruturais das diferentes camadas retinianas - parâmetros considerados como índices de prognóstico e de resposta terapêutica(26-28). Mostra também detalhes reproduzíveis da arquitetura macular e avaliação quantitativa da espessura macular. Como principais causas de BAV tem-se o líquido intrarretiniano e a atrofia isquêmica macular, com consequente aumento e diminuição da espessura vista na OCT, respectivamente.

Nesse estudo os principais achados de OCT foram a membrana epirretineana e a atrofia retiniana, por se tratarem de pacientes crônicos e controlados clinicamente, apresentando líquido intrarretineano em apenas $14,8 \%$ dos casos.

A espessura macular não se correlacionou positivamente com a idade, tempo de doença ou com a AV. Markomichelakis et al. ${ }^{(29)}$, fazem uma revisão de vários artigos que mostram diversos níveis de correlação, fracos e fortes, entre AV e espessura macular. Autores italianos também não encontraram correlação entre espessura macular na OCT e $\mathrm{AV}^{(20)}$. Pesquisadores japoneses correlacionaram a espessura macular com o tempo de doença porém não com a AV ou com a sensibilidade perimétrica da fóvea ${ }^{(30)}$. Uma explicação para essa discrepância entre espessura e AV é a existência de dois tipos de pacientes com DB ocular: um tipo com hipermeabilidade macular associada a vasculite difusa retiniana e aumento de espessura macular; e outro tipo com vasculite oclusiva, atrofia e diminuição da espessura macular. Portanto é infrequente a correlação linear entre espessura macular e AV pois ambos os tipos podem apresentar BAV. Pode-se porém correlacionar fases iniciais da doença com aumento e fases finais com diminuição da espessura macular.

O edema retiniano foi avaliado satisfatoriamente pelos exames de AF e OCT, mostrando índice de concordância em 75\% dos casos com edema confirmado.

Nesse estudo não se encontrou correlação significante entre AV e presença de edema retiniano na AF ou OCT. De acordo com outro trabalho há correlação entre edema macular cistóide e AV, o mesmo não ocorrendo com edema macular difuso(26)

Não se encontrou correlação estatística entre tempo de doença e as variáveis analisadas na OCT.

\section{CONCLUSÃO}

A DB apresenta importante morbidade ocular, com índices significativos de pacientes com visão subnormal ou cegueira legal na sua evolução. Os exames de AF, ICV e OCT fornecem dados importantes do polo posterior para o acompanhamento, terapêutica e prognóstico da doença. Pacientes com aparente controle clínico podem apresentar ainda alterações inflamatórias que, se persistentes, determinam progressão da BAV.

\section{AGRADECIMENTOS}

Agradecemos a Ariadne Stavare Leal, tecnóloga da UNIFESP, que gentilmente realizou os exames de angiografias e tomografias de coerência óptica de nosso estudo.

\section{REFERÊNCIAS}

1. Ehrlich GE. Vasculitis in Behçet's disease. Int Rev Immunol 1997:14:81-8

2. Criteria for diagnosis of Behçet's disease. International Study Group for Behçet's Disease. Lancet. 1990;335(8697):1078-80.

3. Matsumoto T, Uekusa T, Fukuda Y. Vasculo-Behçet's disease: a pathologic study of eight cases. Hum Pathol. 1991;22(1):45-51

4. Tugal-Tutkun I, Onal S, Altan-Yaycioglu R, Huseyin Altunbas H, Urgancioglu M. Uveitis in Behçet disease: an analysis of 880 patients. Am J Ophthalmol. 2004;138(3):373-80.

5. Kitaichi N, Miyazaki A, Iwata D, Ohno S, Stanford MR, Chams H. Ocular features of Behçet's disease: an international collaborative study. Br J Ophthalmol. 2007;91(12):1579-82.

6. Khairallah M, Attia S, Yahia SB, Jenzeri S, Ghrissi R, Jelliti B, et al. Pattern of uveitis in Behçet's disease in a referral center in Tunisia, North Africa. Int Ophthalmol. 2009;29(3):135-41.

7. Ueno H, Takabatake M, Matsuo N. Fluorescein fundus angiography in Behçet's disease: studies on fluorangiographic findings in four types of the disease. Jpn J Clin Ophthalmo 1975;29:45-50.

8. Matsuo T, Sato Y, Shiraga F, Shiragami C, Tsuchida Y. Choroidal abnormalities in Behçet disease observed by simultaneous indocyanine green and fluorescein angiography with scanning laser ophthalmoscopy. Ophthalmology. 1999;106(2):295-300.

9. Bozzoni-Pantaleoni F, Gharbiya M, Pirraglia MP, Accorinti M, Pivetti-Pezzi P. Indocyanine green angiographic findings in Behçet disease. Retina. 2001;21(3):230-6.

10. Atmaca LS, Sonmez PA. Fluorescein and indocyanine green angiography findings in Behçet's disease. Br J Ophthalmol. 2003;87(12):1466-8.

11. Gedik S, Akova Y, Yilmaz G, Bozbeyoglu S. Indocyanine green and fundus fluorescein angiographic findings in patients with active ocular Behçet's disease. Ocul Immuno Inflamm. 2005;13(1):51-8

12. Steel RG, Torrie JH. Principles and procedures of statistics. New York: McGraw-Hill; 1980. p.633.

13. Sakane T, Takeno M, Suzuki N, Inaba G. Behçet's disease. N Engl J Med 1999;341 (17): 1284-91.

14. Ozdal PC, Ortac S, Taskintuna I, Firat E. Posterior segment involvement in ocular Behçet's disease. Eur J Ophthalmol. 2002;12(5):424-31.

15. Yang $P$, Fang W, Meng $Q$, Ren $Y$, Xing L, Kijlstra A. Clinical features of chinese patients with Behçet's disease. Ophthalmology. 2008;115(2):312-8.e4.

16. Klaeger AJ, Tran VT, Hiroz CA, Morisod L, Herbort CP. Use of ultrasound biomicroscopy, indocyanine green angiography and HLA-B51 testing as adjunct methods in the appraisal of Behçet's uveitis. Int Ophthalmol. 2004;25(1):57-63.

17. Krause L, Köhler AK, Altenburg A, Papoutsis N, Zouboulis CC, Pleyer U, et al. Ocular involvement in Adamantiades-Behçet's disease in Berlin, Germany. Graefes Arch Clin Exp Ophthalmol. 2009;247(5):661-6

18. Sáenz-Francés F, Elías-de-Tejada M, Martínez-de-la-Casa JM, Calvo-González C Fernández-Vidal A, Méndez-Hernández C, et al. Ocular inflammatory signs observed in a cohort of Spanish patients with Behçet disease and ocular inflammation. Eur J Ophthalmol. 2008;18(4):563-6.

19. Rohatgi J, Singal A. Ocular manifestations of Behçet's disease in Indian patients. Indian J Ophthalmol. 2003:51(4):309-13.

20. Iaccarino G, Cennamo G, Forte R, Cennamo G. Evaluation of posterior pole with echography and optical coherence tomography in patients with Behçet's disease. Ophthalmologica. 2009:223(4):250-5.

21. Deuter CM, Kötter I, Wallace GR, Murray PI, Stübiger N, Zierhut M. Behçet's disease: ocula effects and treatment. Prog Retin Eye Res. 2008;27(1):111-36.

22. Barra C, Belfort Júnior R, Abreu MT, Kim MK, Martins MC, Petrilli AM. Behçet's disease in Brazil - a review of 49 cases with emphasis on ophthalmic manifestations. Jpn J Ophthalmol. 1991:35(3):339-46. 
23. Frigui M, Kechaou M, Jemal M, Ben Zina Z, Feki J, Bahloul Z. [Optic neuropathy in Behçet's disease: a series of 18 patients]. Rev Med Interne. 2009;30(6):486-91. French.

24. Sungur G, Hazirolan D, Hekimoglu E, Kasim R, Duman S. Late-onset Behçet's disease: demographic, clinical, and ocular features. Graefes Arch Clin Exp Ophthalmol. 2010;248(9): 1325-30.

25. George RK, Chan CC, Whitcup SM, Nussenblatt RB. Ocular immunopathology of Behçet's disease. Surv Ophthalmol. 1997;42(2):157-62.

26. Tran TH, de Smet MD, Bodaghi B, Fardeau C, Cassoux N, Lehoang P. Uveitic macular oedema: correlation between optical coherence tomography patterns with visual acuity and fluorescein angiography. Br J Ophthalmol. 2008;92(7):922-7.

27. Unoki N, Nishijima K, Kita M, Hayashi R, Yoshimura N. Structural changes of fovea during remission of Behçet's disease as imaged by spectral domain optical coherence tomography. Eye (Lond). 2010;24(6):969-75.

28. Sivaprasad S, Ikeji F, Xing W, Lightman S. Tomographic assessment of therapeutic response to uveitic macular oedema. Clin Experiment Ophthalmol. 2007;35(8):719-23.

29. Markomichelakis NN, Halkiadakis I, Pantelia E, Peponis V, Patelis A, Theodossiadis P, et al. Patterns of macular edema in patients with uveitis: qualitative and quantitative assessment using optical coherence tomography. Ophthalmology. 2004;111(5):946-53. Comment in: Ophthalmology. 2005;112(2):360; authorreply 360-1.

30. Takeuchi M, Iwasaki T, Kezuka T, Usui Y, Okunuki Y, Sakai J, et al. Functional and morphological changes in the eyes of Behçet's patients with uveitis. Acta Ophthalmol. 2010;88(2):257-62. 